\title{
The Clinical Significance of Strap Muscle Invasion in Papillary Thyroid Cancer on Local Recurrence: Is Less Surgery Warranted?
}

\author{
Hunter J. Underwood, MD and Kepal N. Patel, MD
}

NYU Langone Health, New York

The most recent American Thyroid Association (ATA) guidelines for the management of differentiated thyroid cancer significantly changed the surgical treatment of papillary thyroid cancer (PTC) by no longer recommending total thyroidectomy for 'low risk' malignancies between 1 and $4 \mathrm{~cm} .{ }^{1,2}$ 'High risk' features are considered to be tumors $>4 \mathrm{~cm}$, the presence of extrathyroidal extension, and/or the presence of lymph node metastasis. The clinical significance of extrathyroidal extension continues to be examined within this paradigm shift, which is reflected in the progressive changes seen in the American Joint Committee on Cancer (AJCC) staging system.

The 5th edition of the AJCC staging system for differentiated thyroid cancer defined evidence of minimal or gross extrathyroidal extension as T4 disease. ${ }^{3}$ This is in stark contrast to the current 8 th edition in which minimal extrathyroidal extension has no impact on $\mathrm{T}$ score, and gross extrathyroidal extension invading other major neck structures (gETE) is distinguished from extrathyroidal extension invading only the strap muscles (strap-gETE). ${ }^{4}$ Despite this distinction, several studies have demonstrated that strap-gETE is not associated with an increased risk of recurrence or decreased survival, which leads to the question of whether strap-gETE alone warrants the designation of a 'high risk' thyroid malignancy. ${ }^{5-7}$ Furthermore,

(C) Society of Surgical Oncology 2019

First Received: 7 August 2019;

Published Online: 30 September 2019

K. N. Patel

e-mail: kepal.patel@nyumc.org if these lesions can be classified as 'low risk', would that suggest that lobectomy would be sufficient for initial surgical management?

For this reason, Song et al. conducted a comprehensive, retrospective cohort study to determine the risk of recurrent disease in patients with PTC with strap-gETE, treated with surgical lobectomy alone. ${ }^{8}$ During the study period, 4793 patients underwent surgery for PTC diagnosed by cytology. Total thyroidectomy was recommended as treatment in accordance with the ATA guidelines, but for those who refused, lobectomy was offered after discussion of the potential risks and benefits. Patients with gross extrathyroidal extension invading perithyroidal organs other than strap muscles, and/or distant metastasis noted preoperatively, were excluded from the study.

Ultimately, 636 patients underwent lobectomy for 1-4 cm PTC, and 50 patients (7.9\%) were noted to have strap-gETE. Patients with strap-gETE were significantly older than those without, however there were no differences in sex, tumor size, multifocality, or presence of lymph node metastasis between groups. Within the median follow-up duration, three patients $(6 \%)$ with strap-gETE developed structural persistent/recurrent disease, which was not significantly different than the 30 patients $(5.1 \%)$ with no gETE who developed structural persistent/recurrent disease. There was no significant difference in diseasefree survival between the groups. The most common site of structural persistence/recurrence was the contralateral lobe in both groups.

Given these results, the authors suggest that lobectomy alone can be performed in PTC with strap-gETE without an increased risk of recurrence, and should be strongly considered given less risk of complications and less need for lifelong thyroid replacement when compared with total thyroidectomy. This consideration is congruent with the 
evidence-based trend of 'less is more' management for well-differentiated thyroid cancer. More importantly, this study encourages re-examination of the ATA risk stratification system and the most current AJCC staging system. which consider strap-gETE to be a poor prognostic sign. Challenges to current guidelines and staging systems are crucial to our understanding of thyroid cancer and are responsible for the evolution in the management of this disease.

\section{REFERENCES}

1. Haugen BR, Alexander EK, Bible KC, et al. 2015 American Thyroid Association management guidelines for adult patients with thyroid nodules and differentiated thyroid cancer: the American Thyroid Association guidelines task force on thyroid nodules and differentiated thyroid cancer. Thyroid. 2016;26(1):1-133.

2. Kim BW, Yousman W, Wong WX, Cheng C, Mcaninch EA. Less is more: comparing the 2015 and 2009 American Thyroid Association guidelines for thyroid nodules and cancer. Thyroid. 2016;26(6):759-764.

3. American Joint Committee on Cancer. Thyroid gland. In: Fleming ID, Cooper JS, Henson DE, et al. (eds). AJCC manual for staging of cancer, 5th Edition. Philadelphia, PA: Lippincott-Raven, 1997. pp. 59-64.

4. Perrier ND, Brierley JD, Tuttle RM. Differentiated and anaplastic thyroid carcinoma: major changes in the American Joint Committee on Cancer Eighth Edition cancer staging manual. CA: A Cancer Journal for Clinicians. 2017;68(1):55-63.

5. Park SY, Kim HI, Kim JH, et al. Prognostic significance of gross extrathyroidal extension invading only strap muscles in differentiated thyroid carcinoma. Br J Surg. 2018;105:1155-1162.

6. Amit M, Boonsripitayanon M, Goepfert RP, et al. Extrathyroidal extension: does strap muscle invasion alone influence recurrence and survival in patients with differentiated thyroid cancer? Ann Surg Oncol. 2018;25:3380-3388.

7. Song E, Lee YM, Oh HS, et al. A relook at the T stage of differentiated thyroid carcinoma with a focus on gross extrathyroidal extension. Thyroid. 2019;29:202-208.

8. Song E, Kim WW, Jeon MJ, et al. Clinical significance of gross invasion of strap muscles in patients with 1- to 4-cm-sized papillary thyroid carcinoma undergoing lobectomy. Ann Surg Oncol. 2019. https://doi.org/10.1245/s10434-019-07778-x.

Publisher's Note Springer Nature remains neutral with regard to jurisdictional claims in published maps and institutional affiliations. 